\title{
Observations of meteor showers with the meteor radar of KFU
}

\author{
S. Kalabanov ${ }^{1}$, D. Korotyshkin ${ }^{1}$, R. Ishmuratov ${ }^{2}$, O. Sherstykov ${ }^{1}$ and \\ F. Valiullin ${ }^{1}$ \\ 1 Kazan Federal University, Russia, Kazan, 16A Kremlevskaya st., office \\ 1404, (E-mail: kazansergei@mail.ru) \\ 2 Kazan State Power Engineering University, Russia, Kazan, 51 \\ Krasnoselskaya st. (E-mail: rash-i@mail.ru)
}

Received: August 1, 2021; Accepted: October 28, 2021

\begin{abstract}
The initial results of a new cycle (since 2016) of meteor observations in Kazan $\left(56^{\circ} \mathrm{N} 49^{\circ} \mathrm{E}\right)$ which is associated with equipping of Kazan Federal University (KFU) with the new meteor radar SKiYMET are given. Simultaneously with the processing of experimental data using the built-in (native) software SKiYMET, the processing was performed using the new software developed in KFU. A comparative analysis showed a significant increase in the number of registrations due to the processing of meteors that are weaker with signal-tonoise ratio. The seasonal and daily dependencies of the meteor number for the period 2016-2019 are given. The obtained distributions of entry meteor speeds and their dependencies by seasons and heights of meteor trails are separately considered. The method of data processing for obtaining coordinates of meteor shower radiants in the equatorial coordinate system is described. The results of observations of the Geminids, Eta Aquariids, Arietids, April Lyrids, Northern and Southern Daytime Omega-Cetids, Daytime Lambda Taurids and Daytime Arietids meteor showers are presented.
\end{abstract}

Key words: Meteor radar - meteor shower - entry meteor speed - radiant coordinates - software

\section{Introduction}

\subsection{Radar equipment and techniques for meteor observations}

Radar observations of meteors in the world have been conducted for more than half a century since the middle of the last century (McKinley \& Millman, 1949). They provide valuable information on the distribution of meteor matter near the Earths orbit. In addition, meteor phenomena are used in the study of the Earths average atmosphere (at heights of $80100 \mathrm{~km}$ ), as well as in radio communications. Radar observations of meteors at Kazan University $\left(56^{\circ} \mathrm{N} 49^{\circ} \mathrm{E}\right)$ began in 1956 and they were held for several decades in a row with a small breaks (Kostylev, 1958). During this time, the Kazan school of researchers was formed (Kalabanov et al., 2018b). One of the last significant scientific advances was the development 
of a discrete quasitomographic method for determining the radiant coordinates of meteor showers. Using this method, it was possible to detect small meteor gathering (showers) and to compute the elements of their astronomical orbits (Sidorov et al., 2005) and (Kalabanov et al., 2018a).

The Kazan Meteor Radar (KMR) is a new generation system deployed on the scientific research area of Kazan Federal University, Tatarstan, Russia $\left(56^{\circ} \mathrm{N}\right.$ $49^{\circ} \mathrm{E}$ ) in March 2015 developed and supplied by the Australian company Genesis Software (http://www.gsoft.com.au/productsandservices/skiymet). KMR is equipped with a $15 \mathrm{~kW}$ (pulse) transmitter with an average power of $1 \mathrm{~kW}$. The carrier frequency of the radar is $29.75 \mathrm{MHz}$. The frequency of the probing pulses is $1594 \mathrm{~Hz}$. The pulse duration is $24 \mu \mathrm{s}$. The basic configuration of the SkiYMET radar has one transmitting antenna (orthogonally dual Yagi 2element antennas) with a circular radiation pattern (Fig.1). As a result there is full radiation coverage of the northern celestial hemisphere.

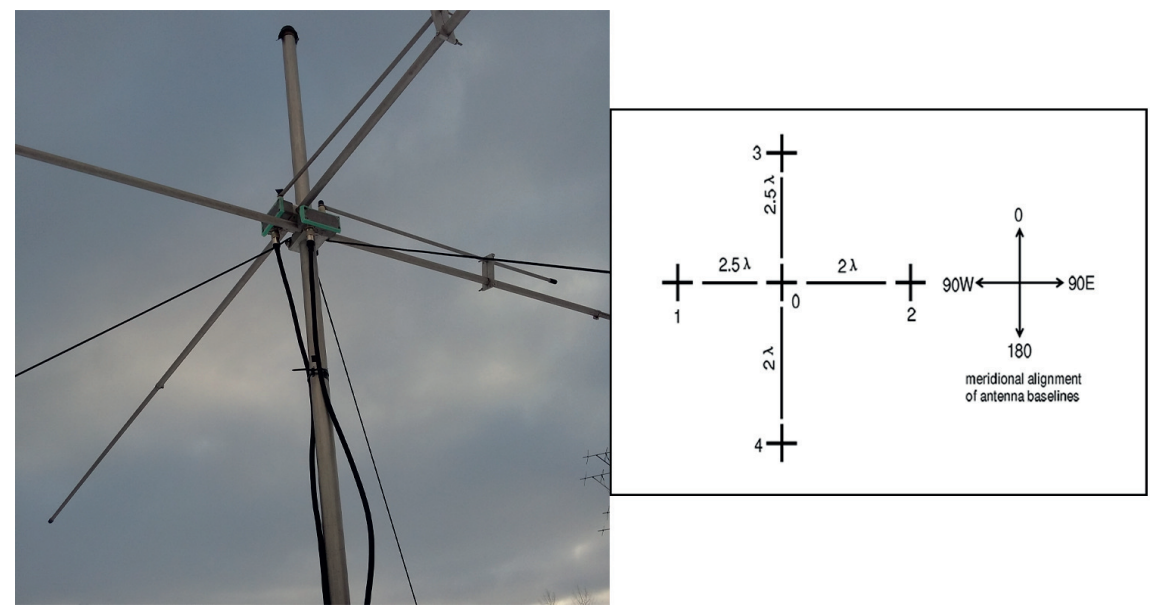

Figure 1. Orthogonally dual Yagi 2-element antennas and antenna system configuration of five antennas $(0,1,2,3,4)$.

The receiving antenna system is a phase interferometer consisting of five antennas arranged in a cross (Fig.1). Each of the five antennas is also orthogonally dual 2-element Yagi antennas with a circular radiation pattern. The phase interferometer makes it possible to determine the angular coordinates of the reflecting points on the meteor tracks. The accuracy parameters for determining the angular coordinates (interferometric errors) depend on the signal-to-noise ratio (SNR) and on the duration of the meteor radio reflection (radio echo). Generally, interferometric errors are below 0.5 degrees, as for other systems based on SKYiMET. The heights of the registered meteor trails range from 80 to $100 \mathrm{~km}$. 
The radar provides observation and computation of the meteor number, the angular coordinates of the reflecting areas on meteor traces, the entry meteor speeds, as well as wind movements of the atmosphere (at heights of meteor traces).

\subsection{Data processing technique}

The method for processing of meteor observation data includes three consecutive stages: 1) primary processing of radar data - measurement of radio reflections and detection (identification) of a meteor; 2) estimation of the entry meteor speed; 3) calculation of the angular coordinates (azimuth and zenith) of the registered meteor radioechos.

After the first years of the radar operation, in addition to the native SKiYMET Genesis Software, we developed our own processing software for primary radar data and meteor detection (KFU software or KFU program). First, the KFU software performs pre-filtering of the primary radar data to eliminate non-meteor reflections of various nature (spark noise, signals reflected from aircrafts, ionosphere backscatter signals). This is provided by step-by-step data processing. In the KFU algorithm, these interfering unwanted objects (spikes and radio reflections from aircraft) are pre-analyzed in the total registration input data and then deleted before final processing (detection of meteor trails). A similar method of input data processing is described in detail in the article (Hocking et al., 2001). Using the new KFU software for input data makes the radar receiving system adaptive to noise environment (interference conditions). As a result, the new filtering algorithm allowed to significantly increase the efficiency of using SKYiMET equipment in terms of detecting meteors, as well as determining meteor parameters.

Secondly, the KFU program allows to estimate entry meteor speed much more efficient (than SKiYMET Software), thanks to the implementation of the Fresnel transform method and the applicable processing of the phase parameters of the radio signal, which we improved according to the works (Hocking, 2000) and (Elford, 2004). The average speed determination accuracy is $3 \mathrm{~km} / \mathrm{s}$.

Thus, we were able to significantly improve the quality and statistical indicators of the processed data. To illustrate this, in Table 1 it is shown the results of a comparative analysis of the effectiveness of data processing with two programs (statistics were collected for the entire observation period from November 2016 to December 2018).

As can be seen from Table 1, the KFU program can detect a much larger number of meteors. In addition, the number of meteors with estimated speeds is about $68 \%$ of the total number of meteor registrations (instead of $6 \%$ for SKiYMET Genesis Software). The achieved result allowed us to conduct more detailed studies of the meteor speed distribution (for different hours of the day and different months). 
Table 1. Comparison of the efficiency of data processing using two programs (statistics are for the entire observation period from November 2016 to December 2018).

\begin{tabular}{|c|c|c|c|}
\hline$\overline{\text { Statistical Data }}$ & $\begin{array}{l}\text { SKiYMET } \\
\text { Genesis } \\
\text { Software }\end{array}$ & $\begin{array}{l}\text { KFU } \\
\text { Software }\end{array}$ & $\begin{array}{l}\text { relation of exceeding } \\
\text { (KFU/Skiymet) }\end{array}$ \\
\hline Total Number of Meteors & 3266901 & 8696502 & 2.66 \\
\hline $\begin{array}{l}\text { Meteor Number } \\
\text { with Estimated Speed }\end{array}$ & 186524 & 5946358 & 31.9 \\
\hline $\begin{array}{l}\text { Percentage of Meteors } \\
\text { with Speed }\end{array}$ & 6 & 68 & \\
\hline
\end{tabular}

The method for determining the angular coordinates (Azimuth and Zenith) of registered meteor radioechoes is described in detail in the article (Jones et al., 1998)

\subsection{The initial main results of meteor observations in 2016-2018}

This paper presents the first initial results of processing experimental data obtained from the new meteor radar. Fig. 2 shows the graphs of daily values of the meteor numbers for the observation cycle 2016-2018 (the processed results are presented in two alternative programs - KFU and SKiYMET programs). Sharp splashes of the curve on the graphs (for individual days) mean days with a partial interruption of observations during the day (due to repair or maintenance work at this time). It can be seen that the number of meteors using KFU program significantly exceeds the number of meteors obtained using the standard SkiYMET software.

Both graphs show a seasonal dependence on the number of meteors (with a maximum in summer and a minimum in early spring) due to the Earth passing through the areas of increased concentration of sporadic meteors distributed in space, as well as meteor showers, in the period from May to November. In addition, against the general background of the number of meteors, there are strong splashes corresponding to the dates of the main meteor showers: Geminids in December (12/16 and 12/17), Quadrantids in January (1/17) and Perseids in August (8/17). Also it can be seen Orionids in mid-October, Eta-Aquariids in late April - early May.

Another important parameter is the speed of meteor particles entering the atmosphere. As we have already noted, the KFU program for determining the entry meteor speed allowed not only to significantly increase the total percentage of recorded meteors while simultaneously determining their speeds (Table 1), but also to better detect meteors with higher speeds $(>50 \mathrm{~km} / \mathrm{s}$, Fig.3). In the presented speed distribution, no correction was made for observational biases (zenith attraction and the effect of Faraday rotation). The factor of the meteor 


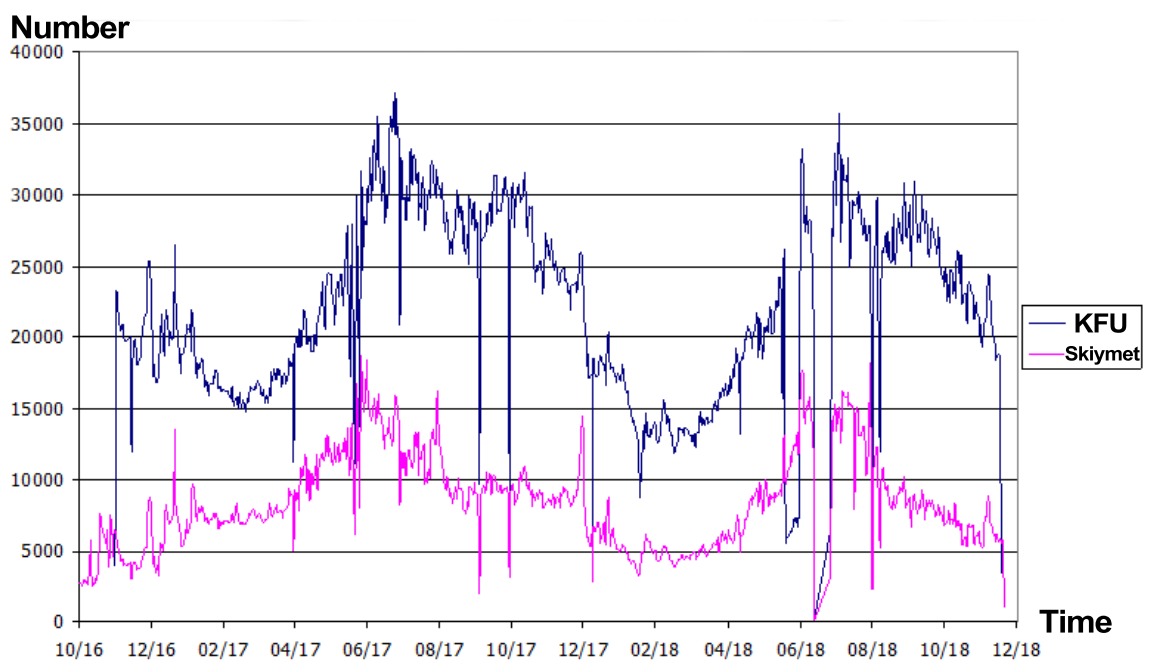

Figure 2. Annual variation of the daily number of meteors in 2016-2018 (detected by KFU and SKiYMET programs)

deceleration was also not taken into account. Since we are dealing with averaged distributions, all of these factors can be neglected here.

As you can see, on the graph of the seasonal dependence of the distribution of meteor speeds, there are two maxima: in the region of $30 \mathrm{~km} / \mathrm{s}$ and $55 \mathrm{~km} / \mathrm{s}$ (Fig.3). In general, during the year meteors prevail with speeds in the range of $20-40 \mathrm{~km} / \mathrm{s}$. In addition, some local maxima occur in January (which corresponds to the Quadrantid shower), as well as in December (35 km/s, Geminids). In Fig. 3 it can be seen that a maximum in the region of $55 \mathrm{~km} / \mathrm{s}$ appears from June to December. Comparison with Fig.2 shows that this is a period of strong meteor activity.

The distributions of speeds and heights (Fig.4) show a rather strong dependence of altitude on the meteor speed. In accordance with the meteor physics, meteors at high speeds begin to burn at high altitudes what is shown on the charts. A local increase in the number of meteors with speeds of about $55 \mathrm{~km} / \mathrm{s}$ is also found in the work of other researchers, for example (Holdsworth et al., 2007). This feature of the speed distribution may be associated with heterogeneity in the distribution of meteor matter in the vicinity of the Earth's orbit. To give a more definitive answer to this question, it is necessary to compare the mutual distribution of meteors by speed and radiant. If high-speed meteors in the speed range of about $55 \mathrm{~km} / \mathrm{s}$ are shown by close radiants, then we can speak of the existence of isolated meteoroid streams. 


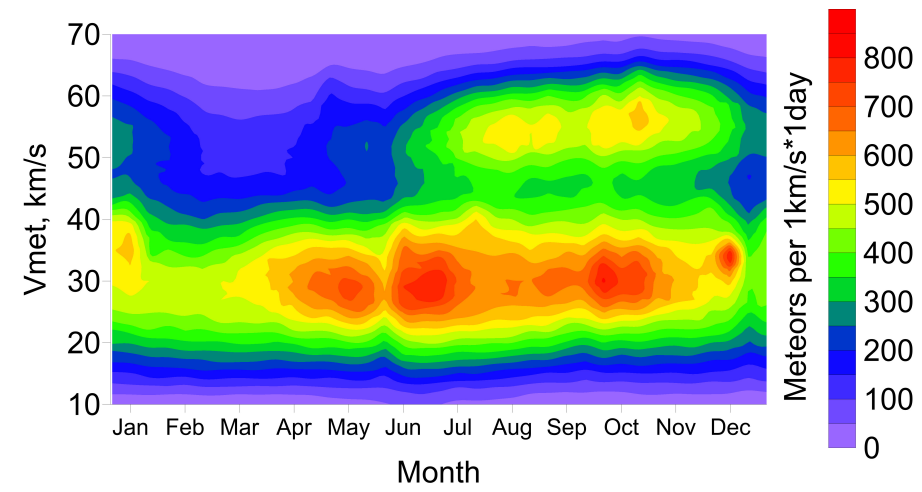

Figure 3. Meteor speed distribution depending on month of the year (for 2017).
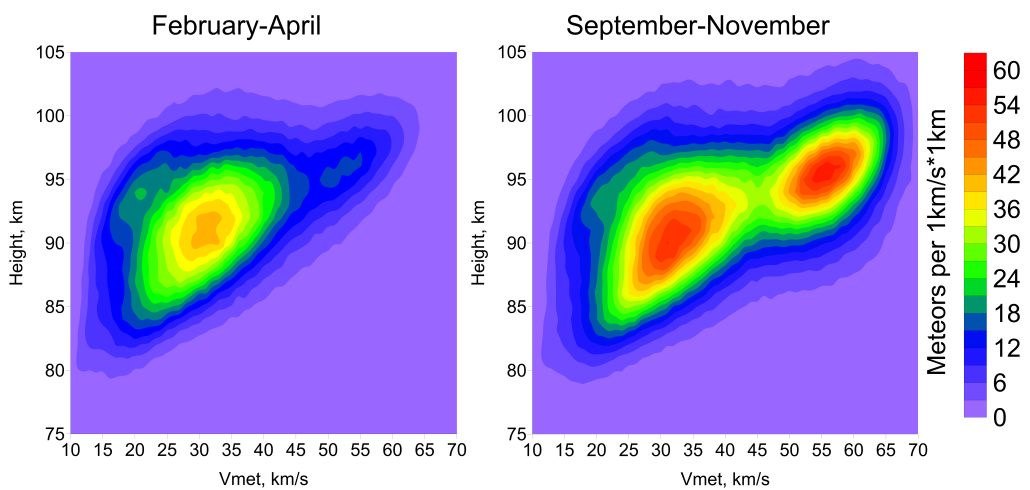

Figure 4. Meteor speed distribution depending on the height (for 2017).

\section{Observations of meteor showers}

\subsection{Method of determination of meteor shower radiants}

The method for determination the coordinates of meteor radiants was borrowed from (Jones \& Jones, 2006). The entire celestial sphere is divided into elementary areas of $2 \times 2$ degrees in the equatorial coordinate system $(\alpha, \delta)$. For each such place it is assigned a unit (test) vector $\mathrm{T}$, which corresponds to the band on the celestial sphere AA (this is the location of points of possible meteor radioechoes) (Fig.5). In fact, the radioecho band GC corresponds to the observed radiant vector $R$. Next, the test vector $T$ closest to the vector $R$ is sought. Then, according to the Jones's method, the integration over the GC and AA bands is performed using the weighting function and as a result, we obtain the response from the transformation system. For the real observed meteor shower 
after integration the response gives the maximum number of meteors and for the sporadic background it gives a minimum number.

To reduce the effect of the presence of a false positive result of the program, we apply the "strip with a positive part" (white stripe) and a "strip with a negative part" (black stripe). In fact, we thereby subtract the sporadic background from the radar response of the meteor shower.


Figure 5. Explanation of the method for determination of meteor shower radiants (figures are taken from the materials of the article (Jones \& Jones, 2006))

Based on this method, a computer program was developed that determines the coordinates of the radiants in the equatorial coordinate system $(\alpha, \delta)$. The input parameters of the program are: the time of the event (radioecho), the angular coordinates of the radio reflection in the horizontal coordinate system (azimuth and zenith), and the entry speed of the meteor relative to the Earth. Fig.6 shows a screenshot of one of the KFU program tabs. Here you can set: sampling intervals by speed, by date, the width of the AA test band, thresholds and other parameters.

Fig.7 shows the distribution of the angular coordinates of radioechoes for one day (December 13, 2018) for the meteor speed range of 30-40 km/s. Shown here: in green - test band AA, corresponding to the previously known coordinates of the Geminid shower radiant, in red - to coordinates of the registered meteor shower radiant per day in the speed range of $30-40 \mathrm{~km} / \mathrm{s}$, in blue - to all meteors per day, including sporadic ones. It can be seen that the data correspond to each other.

After additional transformations, we finally build a 3D distribution of radiant coordinates over the celestial sphere in the equatorial coordinate system, which in this case take the following form (Fig.8). As can be seen, the distribution maximum is concentrated in a narrow region, which corresponds to a meteor shower. In this case, it is the Geminids shower.

Thus, using the Jones's method it is possible to obtain the coordinates of meteor radiants, number of meteors, dates of activity and represent them in the form of a set of three-dimensional graphs depending on speed and time. 


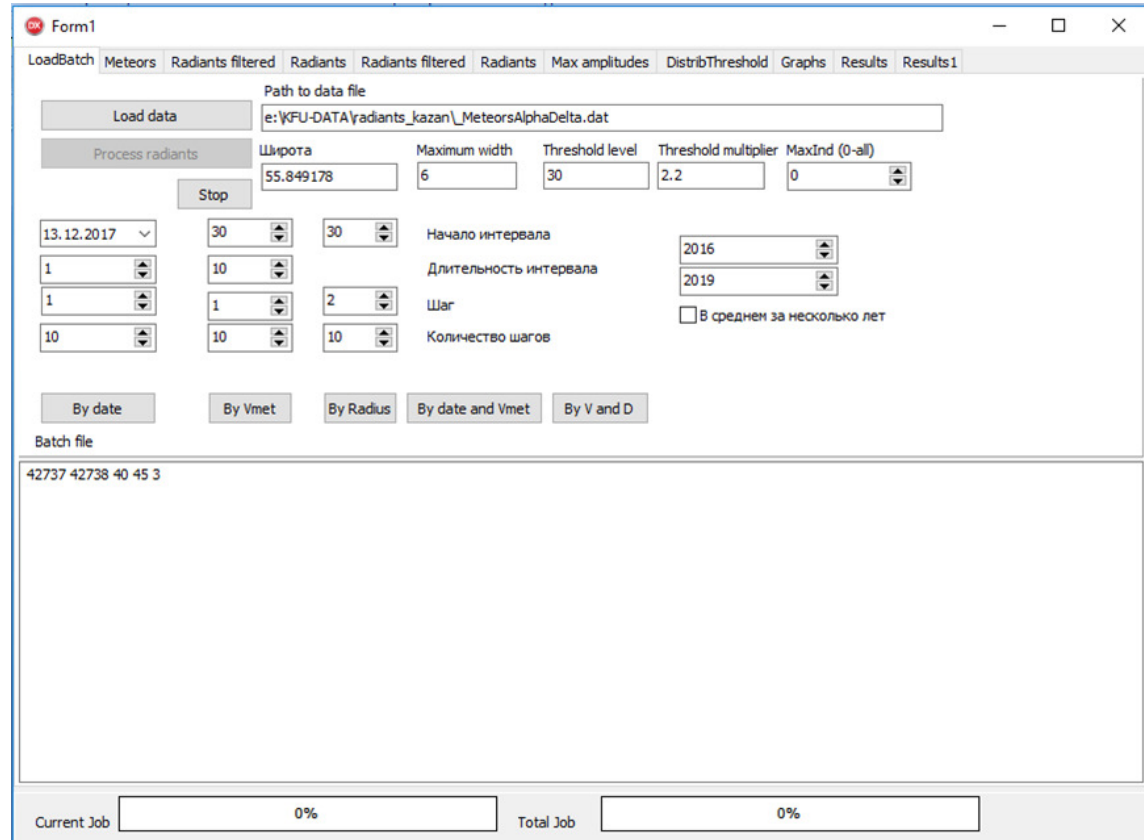

Figure 6. Screenshot of one of the KFU program tabs for determination of radiant coordinates

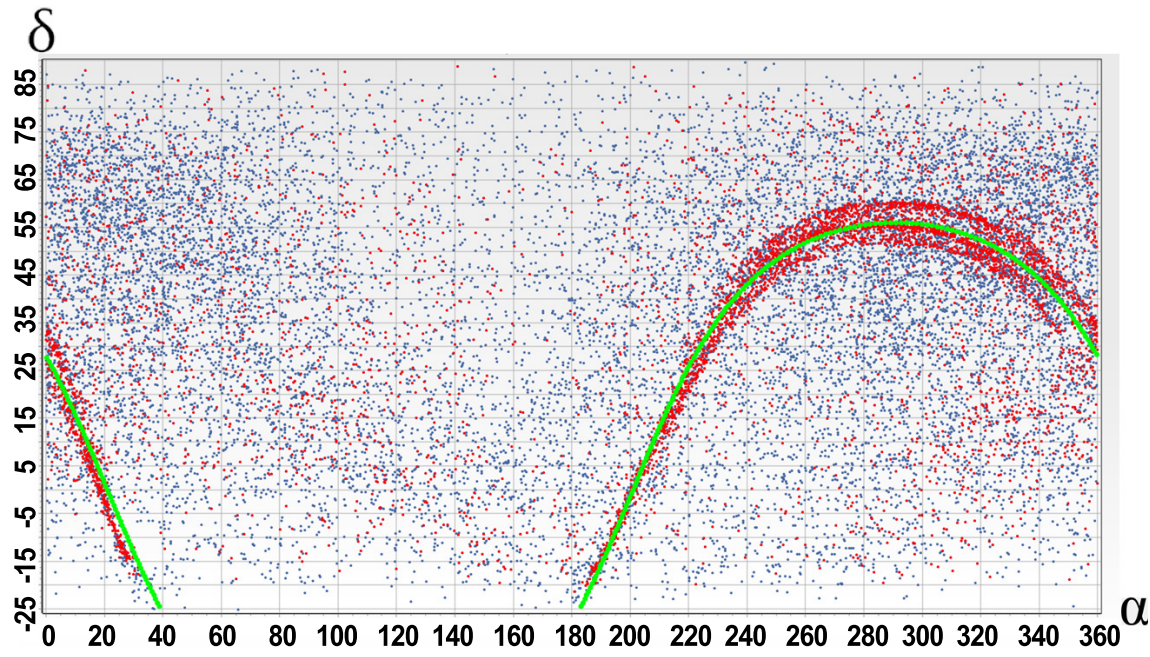

Figure 7. Distribution of angular coordinates of radioechoes in equatorial coordinate system for speed interval of 30-40 km/s for December 13th, 2018 




Figure 8. Distribution of Geminid radiants in equatorial coordinate system for December 13th, 2018

\subsection{Results of observations}

Let's move on to considering the results of observations of meteor showers. Fig.9 shows a summary 3D map of meteor activity for the period from 2017 to 2019. The srong showers are visible - the Quadrantids in January, the Eta-Aquariids in April, the Arietids in June, and the Geminids in December. The Perseids are poorly distinguished in August (which is obviously due to the increased general background of high-speed meteor activity in this area and at this time). All other meteor showers are also visible including the Draconids in 2018.

Fig.10-11 show observational data for the Eta-Aquariids and the Arietids meteor showers in 2017 and 2018. The graphs of changes of the registered number of meteors from day to day are shown, as well as graphs of the gradual daily shift of the radiant coordinates.

\subsection{Brief results of observations of meteor showers in 2019}

Throughout the full year of 2019, the meteor radar was in continuous operation (with the exception of a few hours associated with maintenance). A total of 43 showers were detected, six of which are shown in Fig.12-15. It should be noted that the showers of the southern hemisphere of the celestial sphere, namely the Southern June Aquilids and the Southern Iota Aquariids, were not registered using the KFU radar due to two factors: the location of the radar at northern latitudes $\left(56^{\circ} \mathrm{N} 49^{\circ} \mathrm{E}\right)$ and low shower intensity. At the same time, in the case of sufficiently intense southern showers, such as the Alpha Capricornids, the 


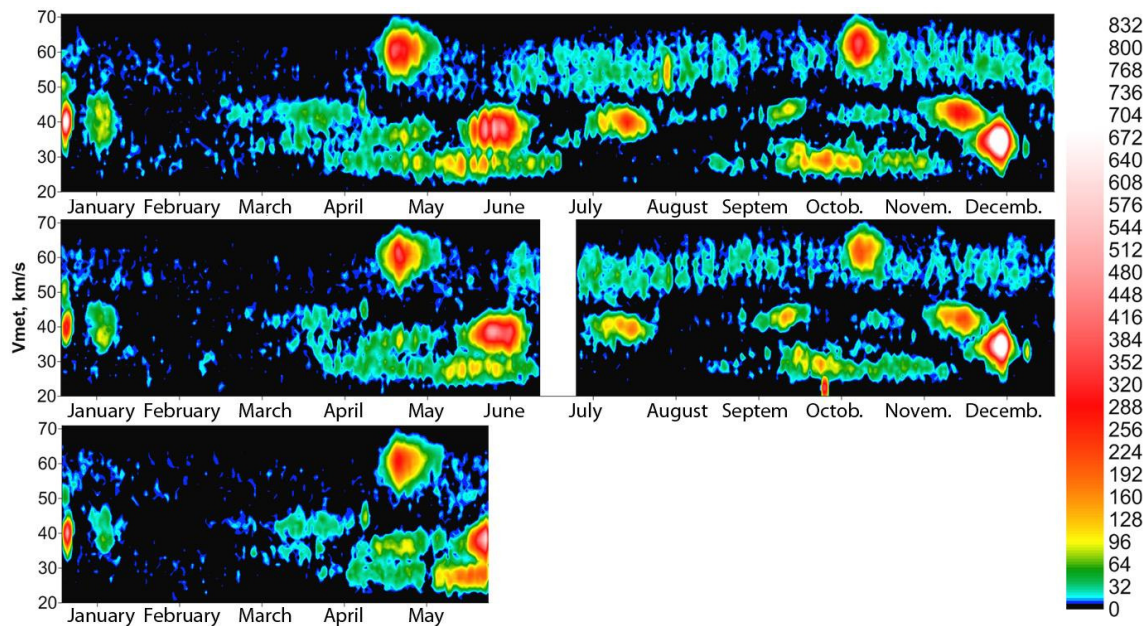

Figure 9. Distribution of meteor showers in 2017-2019
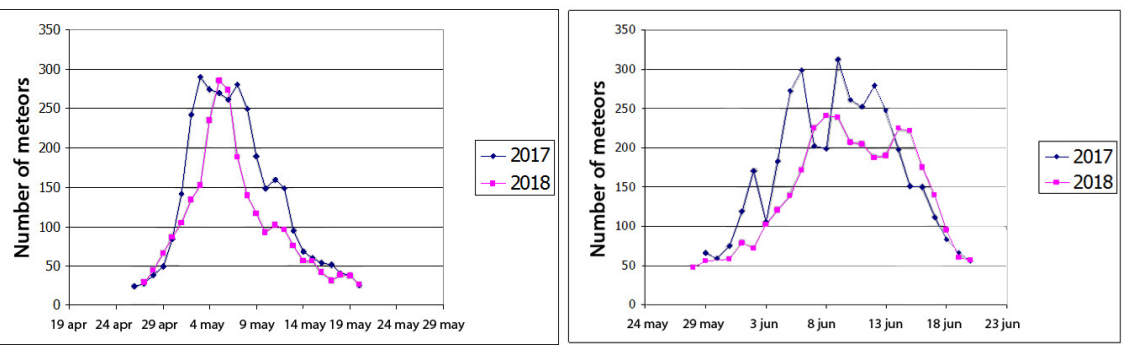

Figure 10. Meteor number from day to day in 2017-2018 (left - Eta Aquariids, right - Arietids)
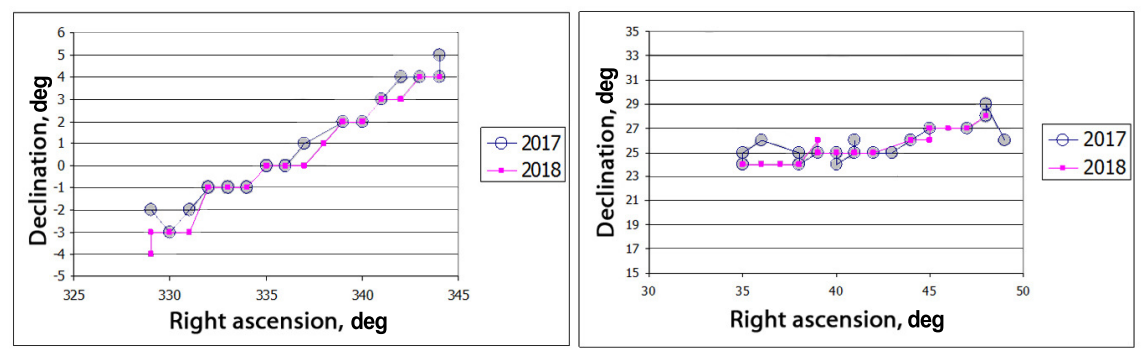

Figure 11. Daily shift of radiant coordinates $(\alpha, \delta)$ in 2017-2018 (left -Eta Aquariids, right - Arietids) 
Southern Delta Aquariids and the Piscis Austrinids the KFU radar was able to register them.

Let's move on to presenting of individual meteor showers. Fig.12-15 show the celestial sphere from $-30^{\circ}$ to $+90^{\circ}$ in declination $(\alpha)$ and from $0^{\circ}$ to $360^{\circ}$ in right ascension $(\delta)$. Fig.13 shows the April Lyrid shower with coordinates $\alpha=273^{\circ}, \delta=34^{\circ}$, speed $46.5 \mathrm{~km} / \mathrm{s}$, date of maximum intensity April 22. This is a low-intensity shower, the number of meteors in 4 days (for a speed range of 44-48 km/s) was 170 meteors. Fig.12 shows data of the Eta Aquariids shower with coordinates $\alpha=339^{\circ}, \delta=0^{\circ}$.

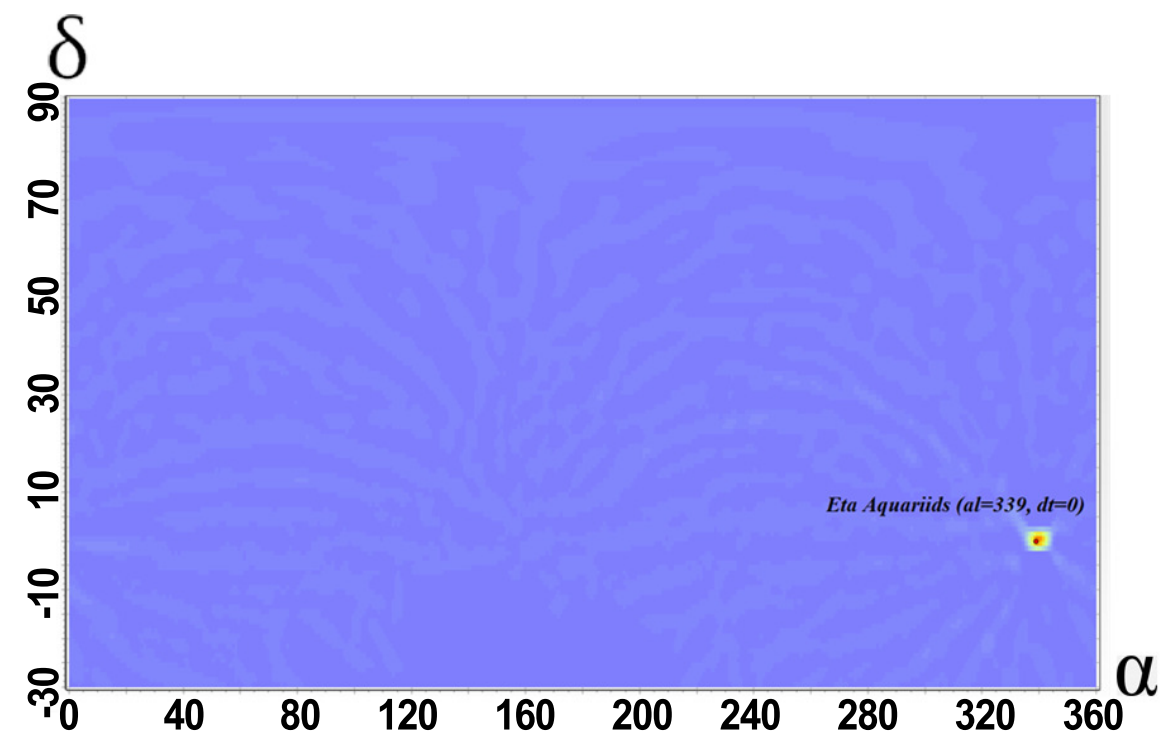

Figure 12. Position of the calculated radiant coordinates for the Eta Aquariids $\left(\alpha=339^{\circ}, \delta=0^{\circ}\right)$ on the celestial sphere in equatorial coordinate system.

Fig.14 shows data of the Northern Daytime Omega-Cetids shower with coordinates $\alpha=12^{\circ}, \delta=20^{\circ}$ and the Southern Daytime Omega-Cetids shower with coordinates $\alpha=23^{\circ}, \delta=-3^{\circ}$. Fig. 15 shows the shower of Daytime Lambda Taurids with coordinates $\alpha=57^{\circ}, \delta=13^{\circ}$. At the same time, in this speed range, it can be seen a more powerful shower of the Daytime Arietids with coordinates $\alpha=48^{\circ}, \delta=28^{\circ}$.

All our data fully correspond to the work of other authors, in particular, to the work (Brown et al., 2008). 


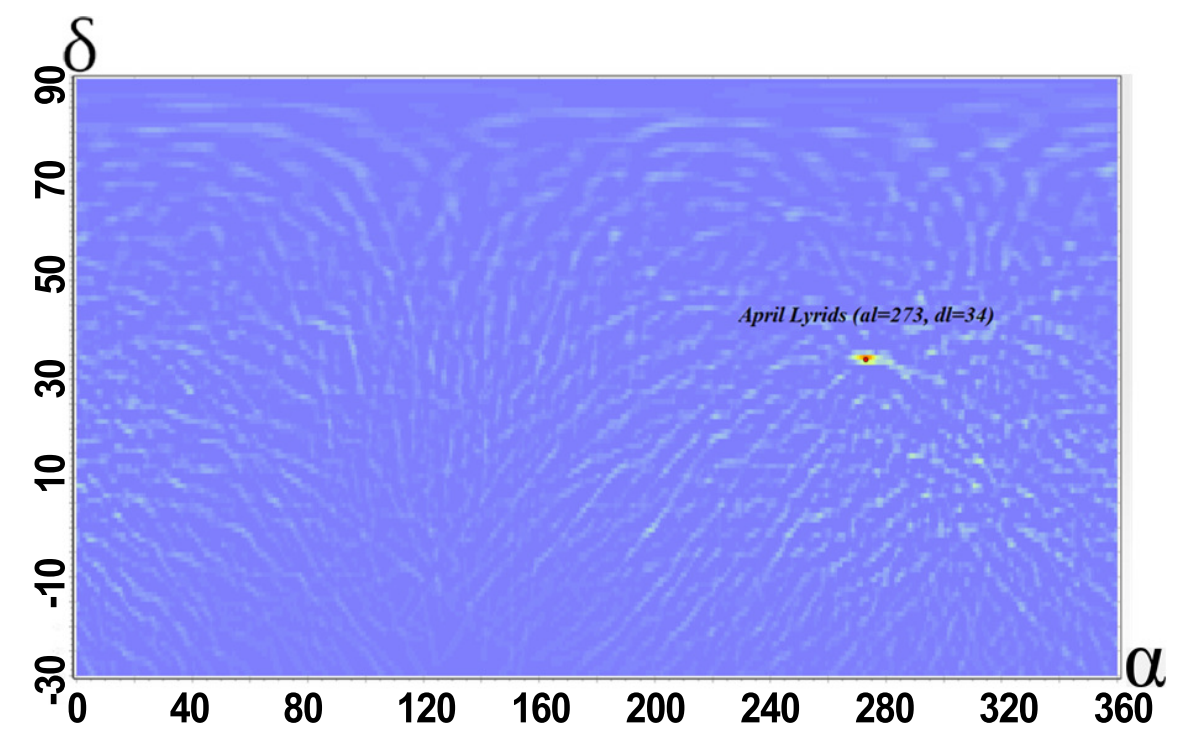

Figure 13. Position of the calculated radiant coordinates for the April Lyrids $\left(\alpha=273^{\circ}, \delta=34^{\circ}\right)$ on the celestial sphere in equatorial coordinate system.

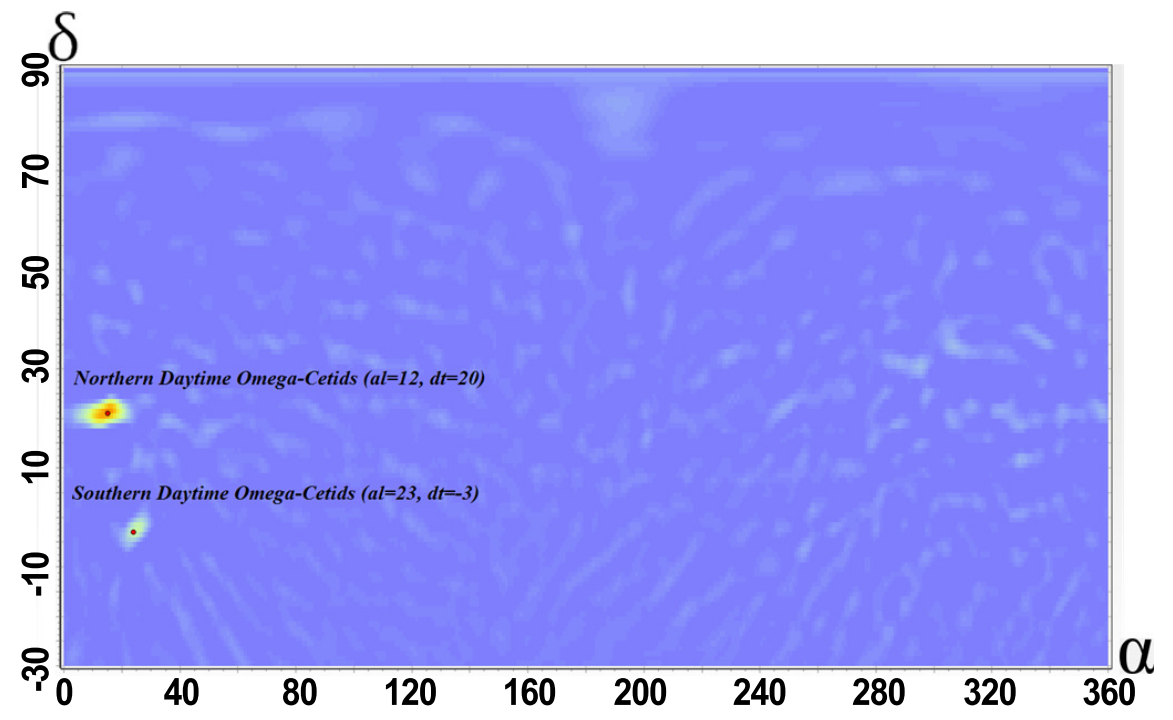

Figure 14. Position of the calculated radiant coordinates for the Northern Daytime Omega-Cetids $\left(\alpha=12^{\circ}, \delta=20^{\circ}\right.$ ) and Southern Daytime Omega-Cetids $\left(\alpha=23^{\circ}, \delta=-3^{\circ}\right)$ on the celestial sphere in equatorial coordinate system. 


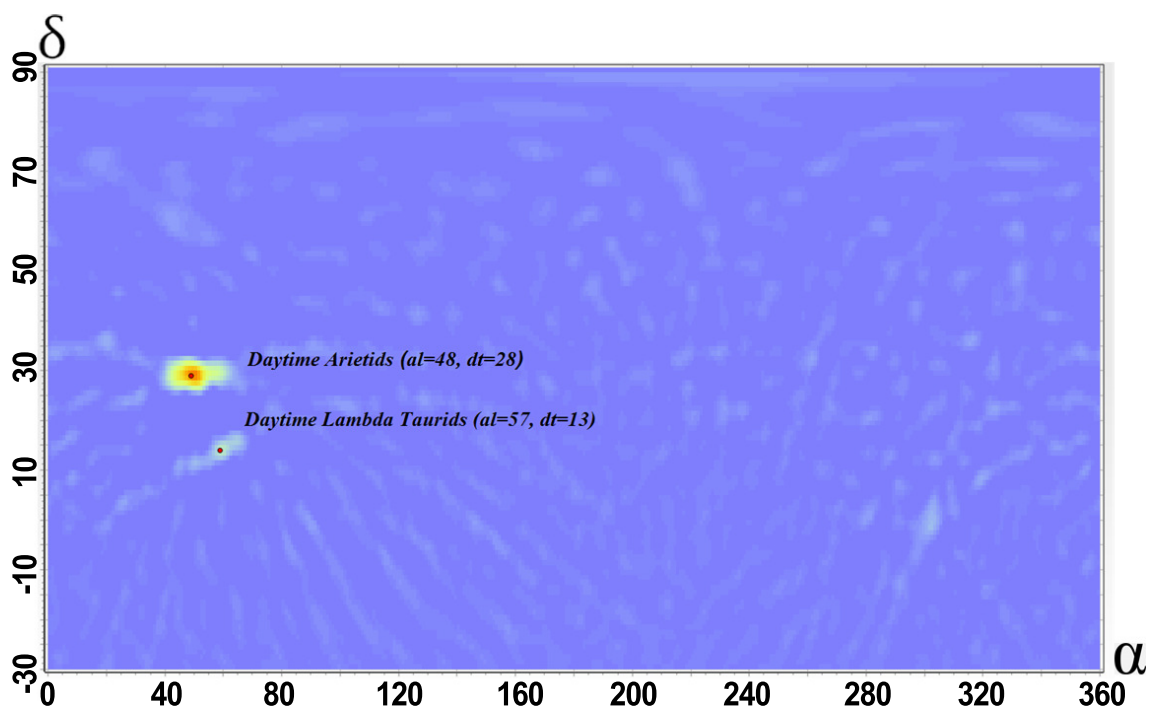

Figure 15. Position of the calculated radiant coordinates for Daytime Lambda Taurids $\left(\alpha=57^{\circ}, \delta=13^{\circ}\right)$ and Daytime Arietids $\left(\alpha=48^{\circ}, \delta=28^{\circ}\right)$ on the celestial sphere in equatorial coordinate system.

\section{Conclusion}

With the equipping of a new meteor radar at Kazan Federal University, a new stage of the long-term meteor observations and scientific researches in Kazan has begun. With the usage of our own algorithm for processing primary meteor registrations, it was possible to significantly improve the quality and statistical indicators of the processed data. Comparative analysis showed a significant increase in the number of registrations due to the processing of meteors with weaker signal-to-noise ratio. The introduction of a new algorithm for determining the speeds of meteors has proven to be especially effective, which makes it possible to perform detailed and statistically secured studies and analysis of experimental data.

The seasonal and daily dependencies of the number and speeds of meteors for the period 2016-2019 as well as the distribution of meteor speeds depending on the heights of meteor trails have been obtained. A total number of 43 meteor showers were registered, their radiant coordinates were calculated. The radiant distribution of the Eta Aquariids, Arietids, April Lyrids, Northern and Southern Daytime Omega-Cetids, Daytime Lambda Taurids and Daytime Arietids are presented. 


\section{Acknowledgements.}

This work was funded by the Russian Government Program of Competitive Growth of Kazan Federal University.

\section{References}

Brown, P., Weryk, R., Wong, D., \& Jones, J., A meteoroid stream survey using the Canadian Meteor Orbit Radar: I. Methodology and radiant catalogue. 2008, Icarus, 195, 317

Elford, W., Radar observations of meteor trails, and their interpretation using Fresnel holography: a new tool in meteor science. 2004, Atmospheric Chemistry and Physics, 4, 911

Hocking, W., Real-time meteor entrance speed determinations made with interferometric meteor radars. 2000, Radio Science, 35, 1205

Hocking, W., Fuller, B., \& Vandepeer, B., Real-time determination of meteor-related parameters utilizing modern digital technology. 2001, Journal of Atmospheric and Solar-Terrestrial Physics, 63, 155

Holdsworth, D., Elford, W., Vincent, R., et al., All-sky interferometric meteor radar meteoroid speed estimation using the Fresnel transform. 2007, in Annales Geophysicae, Copernicus GmbH, 385-398

Jones, J. \& Jones, W., Meteor radiant activity mapping using single-station radar observations. 2006, Monthly Notices of the Royal Astronomical Society, 367, 1050

Jones, J., Webster, A., \& Hocking, W., An improved interferometer design for use with meteor radars. 1998, Radio Science, 33, 55

Kalabanov, S., Sherstyukov, O., Karpov, A., \& Ishmuratov, R., Orbital structure of a meteoric complex in a vicinity of the Earth's orbit by Kazan meteor radar. Types of meteor orbits. 2018a, Planetary and Space Science, 161, 84, DOI: doi.org/10.1016/j.pss.2018.06.015

Kalabanov, S. A., Karpov, A. V., Sulimov, A. I., Sherstyukov, O. N., \& Ishmuratov, R. A., Progress of radar observations of meteors in Kazan (Russia) over the last sixty years. 2018b, Proceedings of the International Astronomical Union, 13, 260, DOI: doi.org/10.1017/S1743921319000401

Kostylev, K. V., Radio equipment of the Engelgardts Astronomical Observatory for automatic observation of meteors (in Russian). 1958, Astronomy Reports, 4, 643

McKinley, D. W. R. \& Millman, P. M., A phenomenological theory of radar echoes from meteors. 1949, Proceedings of the IRE, 37, 364

Sidorov, V., Kalabanov, S., Sidorova, S., Filin, I., \& Filimonova, T., Associations of meteor microshowers or as the Kazan radar SEES radiants on northern celestial hemisphere. 2005, in Modern Meteor Science An Interdisciplinary View (Springer), 165-179 